\title{
Brachial-ankle pulse wave velocity as a risk stratification index for the short-term prognosis of type 2 diabetic patients with coronary artery disease
}

\author{
Michinari Nakamura, Takeshi Yamashita, Junji Yajima, Yuji Oikawa, Koichi Sagara, Akira Koike, \\ Hajime Kirigaya, Kazuyuki Nagashima, Hitoshi Sawada and Tadanori Aizawa, the Shinken Database \\ Study Group
}

The incidence of diabetes is increasing, and the disease has become an important predictor of prognosis in patients with coronary artery disease (CAD), although adverse events often occur without warning. Thus, risk stratification of diabetic CAD patients is important for secondary prevention. This study tests the hypothesis that brachial-ankle pulse wave velocity (baPWV), a marker for arterial stiffness obtained by simple and noninvasive automated devices, can be a risk stratification index to predict prognosis in diabetic patients with CAD. The prognosis of CAD patients with diabetes in the Shinken Database cohort study was investigated by dividing patients into two groups based on baPWV measurements. The composite endpoint was death, nonfatal myocardial infarction, repeat revascularization or readmission for heart failure. Data were available on 564 CAD patients, with a median follow-up of 25.4 months. Of these patients, 191 had type 2 diabetes. The higher baPWV among diabetic patients was defined as a median baPWV of $1730 \mathrm{~cm} \mathrm{~s}^{-1}$ or more. The 3-year Kaplan-Meier estimates of event-free survival were $72.8 \%$ in diabetic patients with lower baPWV and $51.3 \%$ in those with higher baPWV, respectively $(P=0.031)$. Multivariate analysis revealed that a higher baPWV was independently associated with poorer short-term prognosis (hazard ratio, 1.97; 95\% confidence interval, 1.01-3.84) in diabetic CAD patients. In conclusion, baPWV, a marker for arterial stiffness, can be a risk stratification index for short-term prognosis in clinical practice, suggesting the need for further aggressive treatment and strict follow-up in CAD patients with diabetes and higher baPWV.

Hypertension Research (2010) 33, 1018-1024; doi:10.1038/hr.2010.126; published online 12 August 2010

Keywords: arteriosclerosis; brachial-ankle pulse wave velocity; coronary artery disease; diabetes mellitus

\section{INTRODUCTION}

The increasing prevalence of obesity has resulted in an upsurge in the incidence of diabetes mellitus, and consequently a continuing trend toward higher numbers of patients with complications of diabetes. ${ }^{1}$ Diabetes mellitus is a major risk factor for the progression of atherosclerosis and cardiovascular diseases (twice the risk of incident myocardial infarction and stroke as that of the general population) and is also an important predictor for adverse events in patients with coronary artery disease (CAD) ${ }^{2,3}$ Meanwhile, $>30 \%$ of patients with $\mathrm{CAD}$ have diabetes. ${ }^{4}$ Despite the advances in coronary artery bypass grafting and percutaneous coronary intervention, the rate of occurrence of adverse cardiovascular events in diabetic patients is approximately 1.5 - to 2 -fold higher than in nondiabetic patients. ${ }^{5-7}$ Therefore, treatment of diabetic patients with established CAD is crucial; current studies report that intensive glycemic control may be effective early in the course of diabetes, but that this treatment has a minimal or no role in the prognosis of patients with advanced atherosclerosis. ${ }^{8-10}$ The treatment strategy and prognosis among diabetic CAD patients varies, and current guidelines do not risk stratify diabetic patients with established CAD. ${ }^{11-13}$ Identifying a high-risk group among diabetic patients based on the degree of disease control and the duration of the disease is indispensable, but in real clinical practice it is often difficult to determine the duration of diabetes in patients. In addition, adverse cardiovascular events often occur without previous warning in these patients. ${ }^{14-16}$ Accordingly, an improvement in the risk stratification for cardiovascular events in diabetic patients with established CAD is important for secondary prevention, and this measurement needs to be noninvasive and user friendly to facilitate its use in clinical practice.

Arterial stiffening is a manifestation of arteriosclerosis, leading to thickening and loss of elasticity in the arterial wall. One of the indicators of arterial stiffness is pulse wave velocity (PWV), mostly estimated by measuring carotid-femoral PWV. Owing to its time efficiency and technical simplicity, the measurement of brachial-ankle PWV (baPWV) has been proposed and used in the clinical setting. Its validity, reproducibility and high correlation with aortic PWV obtained by a catheter tip manometer have been previously 
reported. ${ }^{17}$ Values of baPWV have been found to correlate with carotid-femoral PWV values ${ }^{18}$ and have also been shown to be significantly associated with the presence and extent of atherosclerotic vascular diseases, ${ }^{19,20}$ cardiovascular risk factors in healthy adolescents, ${ }^{21}$ microalbuminuria and QT interval prolongation in general subjects, ${ }^{22,23}$ and cardiovascular events in patients with heart failure ${ }^{24}$ and acute coronary syndrome. ${ }^{25}$ Moreover, baPWV has been found to be a marker of the efficacy of therapies intended to achieve regression of arterial stiffness. ${ }^{26}$ However, the association between baPWV and the prognosis of $\mathrm{CAD}$ patients with diabetes has not been investigated. Therefore, we hypothesized that baPWV, a marker for arterial stiffness, can be a risk stratification index for prognosis in this population.

The Shinken Database is an ongoing hospital-based epidemiological study designed to assess the mortality and morbidity of Japanese patients with cardiovascular diseases and their risk factors. ${ }^{27-29}$ On the basis of the hypothesis of this study, analyses were conducted in this patient cohort.

\section{METHODS}

\section{Study population}

The Shinken Database study population comprised all new patients who had visited the Cardiovascular Institute Hospital in Tokyo since June 2004 and did not meet any of the exclusion criteria, which included patients with cancer, patients living abroad and patients visiting on a single occasion without any examinations. This study was based on information recorded in the database between June 2004 and March 2007 ( $n=6562)$. Among the subjects included in the database, patients diagnosed as CAD were identified and included in our study $(n=822)$. A total of 212 patients who did not undergo baPWV assessment, and 46 patients with peripheral artery disease (ankle-brachial index $<0.9$ ), aortic aneurysm or atrial fibrillation, were excluded. The remaining 564 patients were included in this study. Type 2 diabetes mellitus was observed in 191 of the patients included in the study. The study population was divided into two groups according to a median baPWV value among diabetic patients $\left(1730 \mathrm{~cm} \mathrm{~s}^{-1}\right)$; the lower baPWV group was defined by a baPWV $<1730 \mathrm{~cm} \mathrm{~s}^{-1}$ and the higher baPWV group was defined by a baPWV of $1730 \mathrm{~cm} \mathrm{~s}^{-1}$ or more.

Written informed consent for inclusion into the Shinken Database was obtained from all participants. The data were transmitted to the Shinken Database center at the hospital, and all clinical outcomes were monitored by medical records, mail and/or telephone contact with the patients and their families.

\section{Measurements of baPWV}

The baPWV was measured using a volume-plethysmographic apparatus (Form/ABI; Colin, Komaki, Japan). Details about this instrument, its use and validation have been described elsewhere. ${ }^{17}$ Briefly, patients were in the supine position and rested for at least $5 \mathrm{~min}$ before measurements were taken. The electrocardiographic electrodes were placed on both wrists and cuffs were wrapped around both brachia and ankles. Pulse volume waveforms at the brachium and ankle were recorded for $10 \mathrm{~s}$ using a semiconductor pressure sensor. Volume waveforms were stored with automatic gain analysis and quality adjustment. The baPWV was automatically calculated according to the following equation:

$$
\mathrm{baPWV}=(\mathrm{La}-\mathrm{Lb}) / T
$$

where $\mathrm{La}$ is the distance from the suprasternal notch to the ankle, $\mathrm{Lb}$ is the distance from the suprasternal notch to the brachium and $T$ is the transit time between the brachial and ankle pulse waves. The highest baPWV on both sides was determined, and subsequent statistical analyses were performed using this value.

\section{Definitions}

Diabetes mellitus was defined by the presence of a history of antidiabetic therapy or as a combination of glycated hemoglobin Alc of $6.5 \%$ or greater and either fasting plasma glucose of $126 \mathrm{mg}$ per $100 \mathrm{ml}$ or greater or 2-h plasma glucose of $200 \mathrm{mg}$ per $100 \mathrm{ml}$ or greater. Body mass index was calculated as the weight in $\mathrm{kg}$ divided by height in $\mathrm{m}$ squared. Hypertension was defined as a systolic blood pressure of $140 \mathrm{~mm} \mathrm{Hg}$ or greater, a diastolic blood pressure of $90 \mathrm{~mm} \mathrm{Hg}$ or greater, or a history of antihypertensive therapy. Hyperuricemia was defined as a plasma uric acid of $7.0 \mathrm{mg}$ per $100 \mathrm{ml}$ or greater. Dyslipidemia was defined as a fasting total cholesterol of $220 \mathrm{mg}$ per $100 \mathrm{ml}$ or more, fasting triglycerides of $150 \mathrm{mg}$ per $100 \mathrm{ml}$ or greater, or a history of lipid-lowering therapy. Renal function was determined by an estimated glomerular filtration rate, calculated by using the glomerular filtration rate equation for Japanese subjects: ${ }^{30}$

$$
\begin{aligned}
\mathrm{GFR}= & 194 \times(\text { serum creatinine })^{-1.094} \times(\text { age })^{-0.287} \\
& \times(0.739 \text { if female })
\end{aligned}
$$

Blood sampling for the baseline lipid and glucose profiles was performed after a 10-12-h fast, although in some patients, especially in those with acute coronary syndrome, blood sampling was performed during the acute phase.

CAD encompasses stable angina pectoris including silent myocardial ischemia, unstable angina, acute myocardial infarction and vasospastic angina. Acute coronary syndrome included unstable angina and acute myocardial infarction. Diagnoses were confirmed by coronary angiogram including fractional flow reserve, noninvasive tests (stress myocardial scintigraphy, treadmill, and stress echocardiography) and/or Holter-ECG. Angiographically significant stenosis was defined as a $50 \%$ or greater stenosis of a major epicardial coronary artery. Angiography findings were checked by more than three coronary interventional cardiologists. Repeat revascularization was defined as percutaneous coronary intervention or coronary artery bypass grafting for the target vessel after the index procedure or revascularization after the decision on medical treatment as a first-line strategy for CAD. Routine follow-up angiography was performed 6 to 9 months after the index procedure and the indication for revascularization was confirmed when patients had apparent myocardial ischemia by noninvasive assessment or in invasive hemodynamic measurements of fractional flow reserve. Information on baseline characteristics of patients, heart function (ejection fraction), their cardiac history, risk factors, the first therapeutic strategies for CAD (percutaneous coronary intervention, coronary artery bypass grafting or medical without invasive treatments) and medications (angiotensin-converting enzyme inhibitors, angiotensin receptor blockers, $\beta$-blockers, statins, Ca channel blockers, aspirin, ticlopidine, cilostazole, nitrate, nicorandil or antidiabetic drugs) at baseline were obtained for all patients.

\section{Study end points}

The primary end point was death from any causes. The secondary end point was the composite of death, nonfatal myocardial infarction, repeat revascularization and readmission for heart failure.

\section{Statistical analysis}

Values were expressed as means \pm s.d. Differences in frequencies were compared by $\chi^{2}$ analysis and differences between mean values were assessed using an unpaired $t$-test. The relationships between baPWV and the estimated glomerular filtration rate or number of diseased vessels were determined by calculations of Pearson's correlation coefficient. Cumulative event curves were plotted by the Kaplan-Meier method stratified according to the presence or absence of diabetes and a higher or lower baPWV; data on patients who were lost to follow-up were censored. The differences in unadjusted survival rates were calculated by log-rank test. The association between the baseline covariates and the outcomes was assessed using a multivariable Cox proportional hazards model. Eleven covariates of clinical relevance thought to impact the outcomes were incorporated into the models: age, gender, presentation (presence or absence of acute myocardial infarction), body mass index, the estimated glomerular filtration rate, presence or absence of hypertension, congestive heart failure on admission, presence or absence of dyslipidemia, use of drug eluting stents, coronary artery bypass grafting and progressive arterial stiffness defined as a baPWV of 1730 or greater. Analyses of data were performed using statistical software (SPSS version 14.0; SPSS Japan, Tokyo, Japan). All $P$-values were two-sided, and significance was defined as $P<0.05$ for all analyses. 


\section{Ethical considerations}

The ethics committee of the institute approved this study and all participants provided written informed consent.

\section{RESULTS}

\section{Baseline characteristics}

Table 1 shows the baseline characteristics of diabetic patients in the higher or lower baPWV groups. The baseline characteristics of nondiabetic patients are also shown as a reference sample. The median follow-up period was 25.4 months (interquartile range 17.5 to 35.4 months). Patients with higher baPWV were older, thinner and had more decreased renal function than those with lower baPWV. The proportions of male gender and dyslipidemia were higher in patients with lower baPWV. Furthermore, hypertension and congestive heart failure on arrival were more frequently observed in the higher baPWV group. There were no significant differences between the two groups in presentation at the initial visit, history of CAD, ejection fraction, lipid values, glycated hemoglobin Alc value and fasting plasma glucose value. Figure 1 shows the correlations between baPWV and renal function or number of diseased vessels in all patients with $\mathrm{CAD}$

Table 1 Baseline characteristics

\begin{tabular}{|c|c|c|c|c|}
\hline \multirow[b]{4}{*}{ Covariates } & \multicolumn{2}{|c|}{$D M(+)$} & \multirow[b]{4}{*}{$P^{*}$} & \multirow{4}{*}{$\begin{array}{l}D M(-) \\
\mathrm{n}=373\end{array}$} \\
\hline & baPWV & baPWV & & \\
\hline & $<1730$ & $\geqslant 1730$ & & \\
\hline & $\mathrm{n}=95$ & $\mathrm{n}=96$ & & \\
\hline Age, years & $60.4 \pm 9.5$ & $70.6 \pm 8.7$ & $<0.001$ & $63.6 \pm 10.6$ \\
\hline Male, $n(\%)$ & $84(88.4)$ & $73(76.0)$ & 0.036 & $299(80.2)$ \\
\hline $\mathrm{BMI}, \mathrm{kg} \mathrm{m}^{-2}$ & $25.0 \pm 3.7$ & $23.9 \pm 3.3$ & 0.030 & $23.9 \pm 3.1$ \\
\hline eGFR, $\mathrm{ml} \mathrm{min}{ }^{-1}$ per $1.73 \mathrm{~m}^{2}$ & $73.6 \pm 20.7$ & $63.2 \pm 23.3$ & 0.001 & $70.5 \pm 19.7$ \\
\hline baPWV, $\mathrm{cm} \mathrm{s}^{-1}$ & $1481 \pm 163$ & $2075 \pm 262$ & $<0.001$ & $1671 \pm 377$ \\
\hline \multicolumn{5}{|l|}{ Presentation, n (\%) } \\
\hline Stable angina & $52(54.7)$ & $57(59.4)$ & & $190(50.9)$ \\
\hline ACS & $38(40.0)$ & $38(39.6)$ & 0.236 & $136(36.5)$ \\
\hline Vasospastic angina & $5(5.3)$ & $1(1.0)$ & & 47 (12.6) \\
\hline Ejection fraction, \% & $61 \pm 15$ & $62 \pm 15$ & 0.708 & $63 \pm 13$ \\
\hline \multicolumn{5}{|l|}{ History of CAD, $\mathrm{n}(\%)$} \\
\hline Previous $\mathrm{PCl}$ & $19(20.0)$ & $19(19.8)$ & 1.000 & $55(14.7)$ \\
\hline Previous CABG & $4(4.2)$ & $7(7.3)$ & 0.537 & $15(4.0)$ \\
\hline Previous MI & $35(36.8)$ & $26(27.1)$ & 0.165 & 89 (23.9) \\
\hline \multicolumn{5}{|l|}{ Comorbidity, n (\%) } \\
\hline Hypertension & $53(55.8)$ & $72(75.0)$ & 0.006 & $212(56.8)$ \\
\hline Hyperuricemia & $15(15.8)$ & $14(14.6)$ & 0.843 & 48 (12.9) \\
\hline Dyslipidemia & $74(77.9)$ & $60(62.5)$ & 0.027 & $230(61.7)$ \\
\hline \multicolumn{5}{|c|}{ Lipid values, mg per $100 \mathrm{ml}$} \\
\hline LDL-cholesterol & $112.9 \pm 27.2$ & $113.3 \pm 34.8$ & 0.924 & $115.3 \pm 31.5$ \\
\hline HDL-cholesterol & $46.8 \pm 12.7$ & $48.3 \pm 10.9$ & 0.414 & $51.3 \pm 15.1$ \\
\hline $\mathrm{HbA} 1 \mathrm{c}, \%$ & $7.2 \pm 1.4$ & $7.3 \pm 1.5$ & 0.501 & $5.3 \pm 0.4$ \\
\hline FPG, mg per $100 \mathrm{ml}$ & $163.4 \pm 62.7$ & $179.3 \pm 70.4$ & 0.107 & $110.2 \pm 21.9$ \\
\hline $\mathrm{CHF}, n(\%)$ & $7(7.4)$ & $20(20.8)$ & 0.012 & $35(9.4)$ \\
\hline
\end{tabular}

Abbreviations: ACS, acute coronary syndrome; baPWV, brachial-ankle pulse wave velocity; $\mathrm{BMI}$, body mass index; CABG, coronary artery bypass grafting; CAD, coronary artery disease; CHF, congestive heart failure; DM, diabetes mellitus; eGFR, estimated glomerular filtration rate FPG, fasting plasma glucose; HbAlc, glycated hemoglobin Alc; HDL, high-density lipoprotein; LDL, low-density lipoprotein; MI, myocardial infarction; PCI, percutaneous coronary intervention. $P^{*}$ baPWV $<1730$ vs. baPWV $\geqslant 1730$ among patients with DM. (a and c) and in diabetic patients with $\mathrm{CAD}$ (b and d). A greater reduction in renal function was significantly associated with increased baPWV in all patients and in diabetic patients. Furthermore, the number of diseased vessels correlated significantly and positively with the baPWV value, and the correlation was stronger when evaluating only diabetic patients.

\section{Therapeutic strategy and medications}

As shown in Table 2, the therapeutic strategies used and medications taken were similar between the two groups, with the exception of $\mathrm{Ca}$ channel blocker, which was more often prescribed to patients with higher baPWV.

\section{Clinical outcomes}

During a median follow-up of 25.4 months, the primary and secondary end points were observed in $2(2.1 \%)$ and $22(23.2 \%)$ patients, respectively, in the diabetic population with lower baPWV, and 10 $(10.4 \%)$ and $37(38.5 \%)$ patients in the diabetic population with higher baPWV (Table 3). The 3-year Kaplan-Meier estimates of survival rates for the diabetic population were $97.8 \%$ for patients with lower baPWV and $85.0 \%$ for patients with higher baPWV (logrank $P=0.019$ ). The 3 -year cardiovascular event-free survival rates in diabetic patients with higher baPWV, lower baPWV and nondiabetic patients were 51.3, 72.8 and $80.8 \%$, respectively (log-rank $P<0.001$ for trend) (Figure 2). The comparison between diabetic patients with higher baPWV and lower baPWV also reached statistical significance ( $\log$-rank $P=0.031$ ), but there were no significant differences in cardiovascular event-free survival between nondiabetic patients and diabetic patients with lower baPWV (log-rank $P=0.141$ ). Thus, the short-term prognosis for diabetic patients with $\mathrm{CAD}$, but without higher baPWV, was similar to that of nondiabetic patients with CAD. The 3-year Kaplan-Meier estimates of cardiovascular event-free survival rate among the nondiabetic CAD patients, who were divided into two groups (the higher and lower baPWV), were also analyzed. Among CAD patients without diabetes, there were no significant differences in cardiovascular event-free survival rates between the higher and lower baPWV group (Supplementary Figure 1). We also evaluated the prognosis by dividing the patients into two groups, namely patients with acute coronary syndrome and stable angina pectoris including vasospastic angina (Supplementary Figure 2).

A multivariate Cox proportional hazard analysis showed that a higher baPWV (the median of baPWV $1730 \mathrm{~cm} \mathrm{~s}^{-1}$ or more) was significantly associated with poorer clinical outcome (hazard ratio 1.97, $95 \%$ confidence interval 1.01 to $3.84, P=0.046$ ) compared with the lower baPWV in the diabetic population with established CAD.

\section{DISCUSSION}

Despite advances in the treatment of cardiovascular diseases, CAD patients with diabetes have poorer clinical outcomes and more frequently suffer adverse cardiovascular events without any symptoms than nondiabetic patients. ${ }^{5-7,14-16}$ Furthermore, the frequency of diabetes among patients with established $\mathrm{CAD}$ is quite high $(30-50 \%) .{ }^{4}$ It is therefore necessary to risk stratify diabetic CAD patients and to treat risk factors for secondary prevention. This study revealed that the extent of arterial stiffness, as measured by the baPWV, was significantly associated with short-term prognosis. The noninvasive, automated and user-friendly nature of baPWV assessment and its ability to improve cardiovascular risk stratification indicates that baPWV could be a useful index for short-term prognosis in clinical practice. On the basis of the baPWV, patients with diabetes and $\mathrm{CAD}$ were reclassified into higher- or lower-risk 

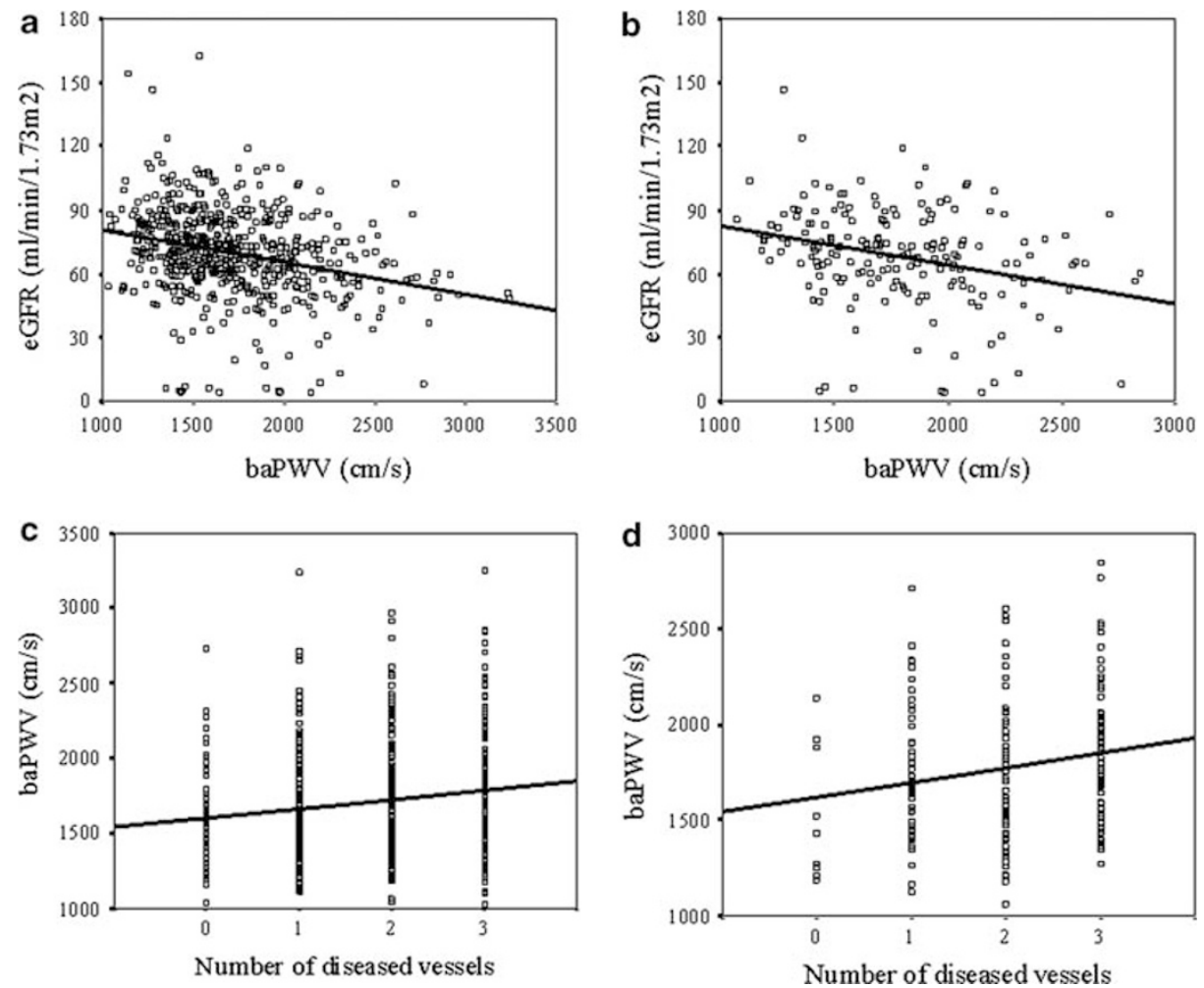

Figure 1 Correlations between brachial-ankle pulse wave velocity (baPWV) and the estimated glomerular filtration rate (eGFR) or number of disease vessels. Correlations between baPWV and eGFR in all patients with coronary artery disease (CAD) (a) $(r=-0.276, P<0.001)$ and diabetic patients with CAD (b) $(r=-0.298, P<0.001)$ and correlations between baPWV and number of diseased vessels in all patients with CAD (c) $(r=0.166, P<0.001)$ and diabetic patients with CAD (d) $(r=0.192, P=0.009)$. Vasospastic angina without significant organic stenosis was classified into zero vessel.

Table 2 Therapy

\begin{tabular}{|c|c|c|c|c|}
\hline \multirow[b]{3}{*}{ Covariates } & \multicolumn{2}{|c|}{$D M(+)$} & \multirow[b]{3}{*}{$\mathrm{P}^{*}$} & \multirow{3}{*}{$\begin{array}{l}D M(-) \\
\mathrm{n}=373\end{array}$} \\
\hline & baPWV & baPWV & & \\
\hline & $\mathrm{n}=95$ & $\mathrm{n}=96$ & & \\
\hline \multicolumn{5}{|l|}{ Therapeutic strategy } \\
\hline CABG, $n(\%)$ & $11(11.6)$ & 15 (15.6) & 0.528 & $38(10.2)$ \\
\hline $\mathrm{PCl}, n(\%)$ & $65(68.4)$ & $66(68.8)$ & 1.000 & $229(61.4)$ \\
\hline Stent, $n(\%)$ & $62(65.3)$ & $62(64.6)$ & 1.000 & 207 (55.5) \\
\hline DES, $n(\%)$ & $42(44.2)$ & $41(42.7)$ & 0.884 & $116(31.1)$ \\
\hline $\mathrm{DCA}, n(\%)$ & $3(3.2)$ & $2(2.1)$ & 0.683 & $28(7.5)$ \\
\hline PTCRA, $n(\%)$ & $5(5.3)$ & $9(9.4)$ & 0.406 & $18(4.8)$ \\
\hline \multicolumn{5}{|l|}{ Medications, n (\%) } \\
\hline ACE inhibitor & $12(12.6)$ & $14(14.9)$ & 0.678 & $61(16.4)$ \\
\hline ARB & $34(35.8)$ & $33(35.1)$ & 1.000 & $105(28.2)$ \\
\hline$\beta$-blocker & $40(42.1)$ & $36(38.3)$ & 0.657 & 134 (35.9) \\
\hline Statin & $57(60.6)$ & $49(52.1)$ & 0.303 & $176(47.2)$ \\
\hline Ca channel blocker & $33(34.7)$ & $49(52.1)$ & 0.019 & $181(48.5)$ \\
\hline Aspirin & $90(94.7)$ & $91(95.8)$ & 1.000 & $324(86.9)$ \\
\hline Ticlopidine & $64(67.4)$ & $72(75.8)$ & 0.260 & $217(58.2)$ \\
\hline Cilostazole & $3(3.2)$ & $4(4.2)$ & 0.722 & $14(3.8)$ \\
\hline Nitrate & $22(23.2)$ & $32(34.0)$ & 0.109 & 78 (20.9) \\
\hline Nicorandil & $32(33.7)$ & $42(44.7)$ & 0.138 & 134 (35.9) \\
\hline
\end{tabular}

Abbreviations: ACE inhibitor, angiotensin-converting enzyme inhibitor; ARB, angiotensin receptor blocker; DES, drug-eluting stent; DCA, directional coronary atherectomy; PTCRA receptor blocker; DES, drug-eluting stent; DCA, directional coronary atherectomy;
percutaneous transluminal coronary rotational ablation. Abbreviations as Table 1 . percutaneous transluminal coronary rotational ablation. Abbreviations as
$P^{*}$ baPWV $<1730$ vs. baPWV $\geqslant 1730 \mathrm{~cm} \mathrm{~s}^{-1}$ among patients with DM.
Table 3 Adverse events

\begin{tabular}{|c|c|c|c|c|}
\hline \multirow[b]{3}{*}{ Events } & \multicolumn{2}{|c|}{$D M(+)$} & \multirow[b]{3}{*}{$\mathrm{P}^{*}$} & \multirow{3}{*}{$\begin{array}{l}D M(-) \\
\mathrm{n}=373\end{array}$} \\
\hline & $\begin{array}{l}\text { baPWV } \\
<1730\end{array}$ & $\begin{array}{l}\text { baPWV } \\
\geqslant 1730\end{array}$ & & \\
\hline & $\mathrm{n}=95$ & $\mathrm{n}=96$ & & \\
\hline The composite end point, $n(\%)$ & $22(23.2)$ & $37(38.5)$ & 0.028 & $63(16.9)$ \\
\hline Death & $2(2.1)$ & $10(10.4)$ & 0.033 & $4(1.1)$ \\
\hline Nonfatal-MI & $0(0)$ & $2(2.1)$ & 0.497 & $4(1.1)$ \\
\hline Revascularization & $20(21.1)$ & $29(30.2)$ & 0.185 & $58(15.5)$ \\
\hline Readmission for heart failure & $2(2.1)$ & $4(4.2)$ & 0.683 & $5(1.3)$ \\
\hline
\end{tabular}

categories. Furthermore, the present findings revealed a need for more aggressive treatment and careful follow-up in CAD patients with both diabetes and advanced arterial stiffness, because this population has a twofold risk for 3-year cardiovascular events.

\section{Coronary atherosclerosis and diabetes}

An earlier population-based autopsy study provided important insights into the relationship between diabetes and coronary atherosclerosis, revealing that among diabetic decedents without clinical $\mathrm{CAD}$, almost three-fourths had high-grade coronary atherosclerosis and more than half had multivessel disease. ${ }^{31}$ Molecular and pathophysiological studies showed that insulin resistance is associated with a pathway-specific impairment in phosphatidylinositol 3-kinase- 


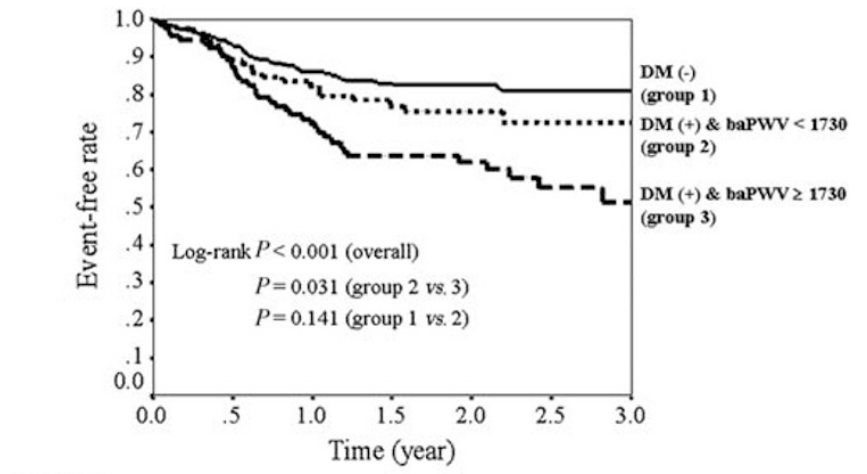

No. at Risk

$\begin{array}{lllll}\text { group 1 } & 373 & 285 & 161 & 76 \\ \text { group 2 } & 95 & 68 & 34 & 11 \\ \text { group 3 } & 96 & 60 & 34 & 11\end{array}$

Figure 2 Kaplan-Meier cardiovascular event-free survival curves. KaplanMeier cardiovascular event-free survival curves based on results obtained with the lower or higher brachial-ankle pulse wave velocity (baPWV) groups (lower than a median baPWV of $1730 \mathrm{~cm} \mathrm{~s}^{-1}$ (group 2) or higher (group 3)) in diabetic patients with coronary artery disease (CAD). Kaplan-Meier cardiovascular event-free survival curve for nondiabetic patients with $C A D$ is also shown, using these patients as a reference group (group 1). The cardiovascular events were defined as the composite of death, nonfatal myocardial infarction, repeat revascularization or readmission for heart failure. DM, diabetes mellitus.

dependent signaling, which may cause an imbalance between the production of nitric oxide and the secretion of endothelin- 1 in the endothelium, decreasing blood flow and leading to endothelial dysfunction. ${ }^{32}$ Meanwhile, hyperglycemia is associated with systemic inflammation and oxidative stress, which may contribute to the accelerated form of atherosclerosis. Impaired endothelial-dependent relaxation $^{33}$ and increasing deposition of fibrous ${ }^{34}$ and calcified tissue $^{35}$ in the arterial wall may, therefore, restrict arterial wall expansion. Furthermore, intravascular ultrasound findings also showed that the presence of diabetes was associated with a greater atherosclerotic burden and impaired compensatory remodeling of the artery wall. ${ }^{36}$ In this study, a higher baPWV was significantly associated with an increasing number of diseased vessels in both CAD patients and CAD patients with diabetes, suggesting that baPWV may reflect the severity of $\mathrm{CAD}$ in the diabetic population. Although the duration and extent of diabetes appear to influence atherosclerosis levels and the prognosis in diabetic CAD patients, this study revealed that the baPWV, an index of arterial stiffness, can predict the prognosis in this population. Without available information on the duration of diabetic history, baPWV can become an alternative tool for risk stratification for short-term outcomes.

\section{CAD and diabetes}

The present results indicate that in CAD patients with both diabetes and higher baPWV, a stricter treatment regimen and more careful follow-up are needed, whereas the short-term prognosis for diabetic patients with established CAD but with lower baPWV was similar to that of nondiabetic CAD patients. The guidelines of the American Diabetes Association, the European Society of Cardiology and the European Association for the Study of Diabetes on diabetes and cardiovascular diseases recommend that a thorough evaluation of coronary, peripheral, renal and cerebrovascular diseases should be performed using repeated measures of inducible ischemia by means of
Holter-ECG, exercise testing, stress echocardiography or myocardial scintigraphy. Moreover, assessment of myocardial viability and left ventricular systolic and diastolic function by means of echo-Doppler and/or magnetic resonance imaging should also be performed. ${ }^{11,13}$ The present results showing that a higher baPWV was significantly associated with a poorer indicate that baPWV should be taken into consideration as a useful index for risk stratification in diabetic CAD patients. In addition, this risk stratification index can become a motivating factor for patients to change their lifestyle and pharmacotherapy to achieve near-normal glycated hemoglobin Alc and to address the modification of other risk factors (for example, physical activity, weight management, blood pressure control and cholesterol management). ${ }^{37,38}$

Many secondary prevention strategies exist to reduce the burden of cardiovascular diseases, including glycemic control and the control of nonglycemic risk factors through blood pressure control, lipid lowering with statin therapy, aspirin therapy, angiotensin-converting enzyme inhibitors, smoking cessation and lifestyle modifications (for example, weight, medical nutrition therapy, physical activity). ${ }^{11,13}$ Intensive glycemic control may be beneficial for the prevention of cardiovascular disease early in the course of type 2 diabetes, but has minimal or no benefit in cases of advanced atherosclerosis. ${ }^{8,10}$ Therefore, intensive glycemic control may not improve the prognosis in diabetic CAD patients with higher baPWV. Intensive statin treatment may regress coronary atherosclerosis, but might not be effective in patients with diabetes. ${ }^{36,39,40}$ There is an ongoing need to develop additional therapies with the aim of reducing the atherosclerotic burden and inhibiting cardiovascular events in CAD patients with both diabetes and advanced arterial stiffness.

The evaluation of the mechanisms underlying the association of baPWV with the prognosis of CAD patients with diabetes was beyond the scope of this study but there are several possible explanations for this association. Arterial stiffening could result in a rise in pulse pressure, causing an increase in the cardiac afterload and a decrease in coronary blood flow, ${ }^{41,42}$ ultimately leading to myocardial ischemia. Also, patients with increased arterial stiffness are more likely to have left ventricular diastolic dysfunction. ${ }^{43}$ Furthermore, the increased arterial stiffness amplifies the pulsatility of arterial blood pressure in fragile capillaries and can lead to organ damage, in particular in high-blood flow, low-resistance organs, such as the brain and kidney. ${ }^{41,42} \mathrm{CAD}$ patients with diabetes show a more significant increase in baPWV than those without diabetes, which could contribute to the association of baPWV, an index of arterial stiffness, with prognosis. Although the mechanisms remain unclear, this study suggests that baPWV can be a risk stratification index in CAD patients with diabetes.

\section{Limitations}

This study has several limitations. First, the present findings were based on a single-center study, and the sample size was relatively small. The present results, therefore, need to be confirmed in multicenter studies with larger populations including other ethnic groups. Furthermore, the study did not include information on the duration of diabetes and lifestyle management with regard to diet and exercise during follow-up. These confounders might influence the prognosis in this population. In addition, blood pressure and baPWV values in this study were obtained on office visits or admission and baPWV values are dependent on blood pressure and its changes, which should have been evaluated before the administration of antihypertensive medication. A lack of morning blood pressure values without antihypertensive medications was therefore an important limitation of the study. 
Hence, the accuracy in the classification of baPWV needs to be considered with caution, and the influence of individual blood pressure changes could not be evaluated accurately. Finally, we investigated the short-term prognosis and the long-term outcomes remain uncertain. However, we believe that risk stratification of shortterm prognosis with baPWV can help target careful follow-up and more intensive medication therapy for patients at higher risk for future cardiovascular events in diabetic patients with established CAD.

In conclusion, our study demonstrated that an increase in arterial stiffness (baPWV $1,730 \mathrm{~cm} \mathrm{~s}^{-1}$ or more) was significantly associated with poorer short-term prognosis, with a hazard ratio of 2.0 compared with lower baPWV in diabetic CAD populations. The present findings suggest that the baPWV, a marker for arterial stiffness obtained by noninvasive and user-friendly devices, may serve as a useful index to identify high-risk groups for short-term prognosis in CAD patients with diabetes in clinical practice.

\section{CONFLICT OF INTEREST}

The authors declare no conflict of interest.

\section{ACKNOWLEDGEMENTS}

We thank Mr Morito Fukuda and Drs Ken Ogasawara, Tokuhisa Uejima, Takayuki Otsuka, Ayumi Goda, Ryuichi Funada and Shunsuke Matsuno of the Cardiovascular Institute and Dr Shinya Suzuki of the University of Tokyo hospital for data collection. We also thank all study participants, physicians, co-medical staff and co-workers.

1 Fox CS, Pencina MJ, Meigs JB, Vasan RS, Levitzky YS, D'Agostino Sr RB. Trends in the incidence of type 2 diabetes mellitus from the 1970s to the 1990s: the Framingham Heart Study. Circulation 2006; 113: 2914-2918.

2 Gu K, Cowie CC, Harris MI. Mortality in adults with and without diabetes in a nationa cohort of the U.S. population, 1971-1993. Diabetes Care 1998; 21: 1138-1145.

3 Huxley R, Barzi F, Woodward M. Excess risk of fatal coronary heart disease associated with diabetes in men and women: meta-analysis of 37 prospective cohort studies. $\mathrm{Br}$ Med J 2006; 332: 73-78.

4 Shaw LJ, Shaw RE, Merz CN, Brindis RG, Klein LW, Nallamothu B, Douglas PS, Krone RJ, McKay CR, Block PC, Hewitt K, Weintraub WS, Peterson ED, American College of Cardiology-National Cardiovascular Data Registry Investigators. Impact of ethnicity and gender differences on angiographic coronary artery disease prevalence and in-hospital mortality in the American College of Cardiology-National Cardiovascular Data Registry. Circulation 2008; 117: 1787-1801.

5 lijima R, Ndrepepa G, Mehilli J, Markwardt C, Bruskina O, Pache J, Ibrahim M, Schömig A, Kastrati A. Impact of diabetes mellitus on long-term outcomes in the drug-eluting stent era. Am Heart J 2007; 154: 688-693.

6 Fröbert 0 , Lagerqvist B, Carlsson J, Lindbäck J, Stenestrand U, James SK. Differences in restenosis rate with different drug-eluting stents in patients with and without diabetes mellitus: a report from the SCAAR (Swedish Angiography and Angioplasty Registry). J Am Coll Cardiol 2009; 53: 1660-1667.

7 Brener SJ, Lytle BW, Casserly IP, Schneider JP, Topol EJ, Lauer MS. Propensity analysis of long-term survival after surgical or percutaneous revascularization in patients with multivessel coronary artery disease and high-risk features. Circulation 2004; 109: 2290-2295.

8 Holman RR, Paul SK, Bethel MA, Matthews DR, Neil HA. 10-year follow-up of intensive glucose control in type 2 diabetes. N Engl J Med 2008; 359: 1577-1589.

9 Nathan DM, Cleary PA, Backlund JY, Genuth SM, Lachin JM, Orchard TJ, Raskin P, Zinman B, Diabetes Control and Complications Trial/Epidemiology of Diabetes Interventions Complications (DCCT/EDIC) Study Research Group. Intensive diabetes treatment and cardiovascular disease in patients with type 1 diabetes. N Engl J Med 2005; 353: 2643-2653.

10 Skyler JS, Bergenstal R, Bonow RO, Buse J, Deedwania P, Gale EA, Howard BV, Kirkman MS, Kosiborod M, Reaven P, Sherwin RS, American Diabetes Association; American College of Cardiology Foundation; American Heart Association. Intensive glycemic control and the prevention of cardiovascular events: implications of the ACCORD, ADVANCE, and VA diabetes trials: a position statement of the American Diabetes Association and a scientific statement of the American College of Cardiology Foundation and the American Heart Association. Circulation 2009; 119: 351-357.

11 American Diabetes Association. Standards of medical care in diabetes: 2008. Diabetes Care 2008; 31(Suppl1): S12-S54

12 Buse JB, Ginsberg HN, Bakris GL, Clark NG, Costa F, Eckel R, Fonseca V, Gerstein HC Grundy S, Nesto RW, Pignone MP, Plutzky J, Porte D, Redberg R, Stitzel KF, Stone NJ, American Heart Association; American Diabetes Association. Primary prevention of cardiovascular diseases in people with diabetes mellitus: a scientific statement from the American Heart Association and the American Diabetes Association. Circulation 2007; 115: 114-126.

13 Rydén L, Standl E, Bartnik M, Van den Berghe G, Betteridge J, de Boer MJ, Cosentino F, Jönsson B, Laakso M, Malmberg K, Priori S, Ostergren J, Tuomilehto J, Thrainsdottir I, Vanhorebeek I, Stramba-Badiale M, Lindgren P, Qiao Q, Priori SG, Blanc JJ, Budaj A, Camm J, Dean V, Deckers J, Dickstein K, Lekakis J, McGregor K, Metra M, Morais J, Osterspey A, Tamargo J, Zamorano JL, Deckers JW, Bertrand M, Charbonnel B, Erdmann E, Ferrannini E, Flyvbjerg A, Gohlke H, Juanatey JR, Graham I, Monteiro PF, Parhofer K, Pyörälä K, Raz I, Schernthaner G, Volpe M, Wood D, Task Force on Diabetes Cardiovascular Diseases of the European Society of Cardiology (ESC); European Association for the Study of Diabetes (EASD. Guidelines on diabetes, pre-diabetes, and cardiovascular diseases: executive summary. The Task Force on Diabetes and Cardiovascular Diseases of the European Society of Cardiology (ESC) and of the European Association for the Study of Diabetes (EASD). Eur Heart J 2007; 28: 88-136.

14 Myerburg RJ, Kessler KM, Castellanos A. Sudden cardiac death: epidemiology, transient risk, and intervention assessment. Ann Intern Med 1993; 119: 1187-1197.

15 Stecker EC, Vickers C, Waltz J, Socoteanu C, John BT, Mariani R, McAnulty JH, Gunson K, Jui J, Chugh SS. Population-based analysis of sudden cardiac death with and without left ventricular systolic dysfunction: two-year findings from the Oregon Sudden Unexpected Death Study. J Am Coll Cardiol 2006; 47: 1161-1166.

16 Chugh SS, Reinier K, Singh T, Uy-Evanado A, Socoteanu C, Peters D, Mariani R, Gunson K, Jui J. Determinants of prolonged QT interval and their contribution to sudden death risk in coronary artery disease: the Oregon Sudden Unexpected Death Study. Circulation 2009; 119: 663-670.

17 Yamashina A, Tomiyama H, Takeda K, Tsuda H, Arai T, Hirose K, Koji Y, Hori S, Yamamoto $Y$. Validity, reproducibility, and clinical significance of noninvasive brachialankle pulse wave velocity measurement. Hypertens Res 2002; 25: 359-364.

18 Tanaka H, Munakata M, Kawano $Y$, Ohishi M, Shoji T, Sugawara J, Tomiyama H, Yamashina A, Yasuda H, Sawayama T, Ozawa T. Comparison between carotid-femoral and brachial-ankle pulse wave velocity as measures of arterial stiffness. $J$ Hypertens 2009; 27: 2022-2027.

19 Yamashina A, Tomiyama H, Arai T, Hirose K, Koji Y, Hirayama Y, Yamamoto Y, Hori S. Brachial-ankle pulse wave velocity as a marker of atherosclerotic vascular damage and cardiovascular risk. Hypertens Res 2003; 26: 615-622.

20 Imanishi R, Seto S, Toda G, Yoshida M, Ohtsuru A, Koide Y, Baba T, Yano K. High brachial-ankle pulse wave velocity is an independent predictor of the presence of coronary artery disease in men. Hypertens Res 2004; 27: 71-78.

21 Im JA, Lee JW, Shim JY, Lee HR, Lee DC. Association between brachial-ankle pulse wave velocity and cardiovascular risk factors in healthy adolescents. J Pediatr 2007. 150: 247-251

22 Ishikawa T, Hashimoto J, Morito RH, Hanazawa T, Aikawa T, Hara A, Shintani Y, Metoki $\mathrm{H}$, Inoue $\mathrm{R}$, Asayama $\mathrm{K}$, Kikuya $\mathrm{M}$, Ohkubo $\mathrm{T}$, Totsune $\mathrm{K}$, Hoshi $\mathrm{H}$, Satoh $\mathrm{H}$, Imai $\mathrm{Y}$. Association of microalbuminuria with brachial-ankle pulse wave velocity: the Ohasama study. Am J Hypertens 2008; 21: 413-418.

23 Maebuchi D, Arima H, Ninomiya T, Yonemoto K, Kubo M, Doi Y, Tanizaki Y, Matsumura K, lida M, Kiyohara Y. Arterial stiffness and QT interval prolongation in a general population: the Hisayama study. Hypertens Res 2008; 31: 1339-1345.

24 Meguro T, Nagatomo Y, Nagae A, Seki C, Kondou N, Shibata M, Oda Y. Elevated arterial stiffness evaluated by brachial-ankle pulse wave velocity is deleterious for the prognosis of patients with heart failure. Circ J 2009; 73: 673-680.

25 Tomiyama H, Koji Y, Yambe M, Shiina K, Motobe K, Yamada J, Shido N, Tanaka N, Chikamori T, Yamashina A. Brachial—ankle pulse wave velocity is a simple and independent predictor of prognosis in patients with acute coronary syndrome. Circ J 2005; 69: 815-822.

26 Gando Y, Kawano H, Yamamoto K, Sanada K, Tanimoto M, Oh T, Ohmori Y, Miyatani M, Usui C, Takahashi E, Tabata I, Higuchi M, Miyachi M. Age and cardiorespiratory fitness are associated with arterial stiffening and left ventricular remodelling. J Hum Hypertens 2010; 24: 197-206.

27 Nakamura M, Yamashita T, Yajima J, Oikawa Y, Ogasawara K, Kirigaya H, Sagara K, Koike A, Sawada H, Aizawa T. Impact of reduced renal function on prognosis in Japanese patients with coronary artery disease: a prospective cohort of Shinken database 2007. Hypertens Res 2009; 32: 920-926.

28 Nakamura M, Yamashita T, Yajima J, Oikawa Y, Ogasawara K, Kirigaya H, Nagashima K, Sawada H, Aizawa T. Long-term safety and efficacy of sirolimus-eluting stents in Japanese patients: a single-center cohort study. J Invasive Cardiol 2009; 21: 526-531.

29 Nakamura M, Yamashita T, Yajima J, Oikawa Y, Ogasawara K, Sagara K, Kirigaya H, Koike A, Nagashima K, Ohtsuka T, Uejima T, Suzuki S, Sawada H, Aizawa T. Clinical outcome after acute coronary syndrome in Japanese patients: an observational cohort study. J Cardiol 2010; 55: 69-76.

30 Matsuo S, Imai E, Horio M, Yasuda Y, Tomita K, Nitta K, Yamagata K, Tomino Y, Yokoyama $\mathrm{H}$, Hishida $\mathrm{A}$. Revised equations for estimated GFR from serum creatinine in Japan. Am J Kidney Dis 2009; 53: 982-992.

31 Goraya TY, Leibson CL, Palumbo PJ, Weston SA, Killian JM, Pfeifer EA, Jacobsen SJ, Frye RL, Roger VL. Coronary atherosclerosis in diabetes mellitus: a population-based autopsy study. J Am Coll Cardiol 2002; 40: 946-953.

32 Kim JA, Montagnani M, Koh KK, Quon MJ. Reciprocal relationships between insulin resistance and endothelial dysfunction: molecular and pathophysiological mechanisms. Circulation 2006; 113: 1888-1904.

33 Cersosimo E, DeFronzo RA. Insulin resistance and endothelial dysfunction: the road map to cardiovascular diseases. Diabetes Metab Res Rev 2006; 22: 423-436. 
34 Portik-Dobos V, Anstadt MP, Hutchinson J, Bannan M, Ergul A. Evidence for a matrix metalloproteinase induction/activation system in arterial vasculature and decreased synthesis and activity in diabetes. Diabetes 2002; 51: 3063-3068.

35 Anand DV, Lim E, Darko D, Bassett P, Hopkins D, Lipkin D, Corder R, Lahiri A. Determinants of progression of coronary artery calcification in type 2 diabetes role of glycemic control and inflammatory/vascular calcification markers. J Am Coll Cardiol 2007; 50: 2218-2225.

36 Nicholls SJ, Tuzcu EM, Kalidindi S, Wolski K, Moon KW, Sipahi I, Schoenhagen P, Nissen SE. Effect of diabetes on progression of coronary atherosclerosis and arterial remodeling: a pooled analysis of 5 intravascular ultrasound trials. J Am Coll Cardiol 2008; 52: 255-262.

37 Smith Jr SC, Allen J, Blair SN, Bonow RO, Brass LM, Fonarow GC, Grundy SM, Hiratzka L, Jones D, Krumholz HM, Mosca L, Pasternak RC, Pearson T, Pfeffer MA, Taubert KA, AHA/ACC; National Heart, Lung, Blood Institute. AHA/ACC guidelines for secondary prevention for patients with coronary and other atherosclerotic vascular disease: 2006 update: endorsed by the National Heart, Lung, and Blood Institute. Circulation 2006; 113: 2363-2372.

38 Marwick TH, Hordern MD, Miller T, Chyun DA, Bertoni AG, Blumenthal RS, Philippides G, Rocchini A, Council on Clinical Cardiology, American Heart Association Exercise, Cardiac Rehabilitation, and Prevention Committee; Council on Cardiovascular Disease in the Young; Council on Cardiovascular Nursing; Council on Nutrition, Physical
Activity, and Metabolism; Interdisciplinary Council on Quality of Care and Outcomes Research. Exercise training for type 2 diabetes mellitus: impact on cardiovascular risk: a scientific statement from the American Heart Association. Circulation 2009; 119: 3244-3262.

39 Nissen SE, Tuzcu EM, Schoenhagen P, Brown BG, Ganz P, Vogel RA, Crowe T, Howard G, Cooper CJ, Brodie B, Grines CL, DeMaria AN. Effect of intensive compared with moderate lipid-lowering therapy on progression of coronary atherosclerosis: a randomized controlled trial. JAMA 2004; 291: 1071-1080.

40 Nissen SE, Nicholls SJ, Sipahi I, Libby P, Raichlen JS, Ballantyne CM, Davignon J, Erbel R, Fruchart JC, Tardif JC, Schoenhagen P, Crowe T, Cain V, Wolski K, Goormastic M, Tuzcu EM. Effect of very high-intensity statin therapy on regression of coronary atherosclerosis: the ASTEROID trial. JAMA 2006; 295: 1556-1565.

41 Tomiyama H, Yamashina A. Non-invasive vascular function tests: their pathophysiological background and clinical application. Circ J 2010; 74: 24-33.

42 Wilkinson IB, McEniery CM, Cockcroft JR. Arteriosclerosis and atherosclerosis: guilty by association. Hypertension 2009; 54: 1213-1215.

43 Su HM, Lin TH, Lee CS, Lee HC, Chu CY, Hsu PC, Voon WC, Lai WT, Sheu SH. Myocardial performance index derived from brachial-ankle pulse wave velocity: a novel and feasible parameter in evaluation of cardiac performance. Am J Hypertens 2009; 22: 871-876.

Supplementary Information accompanies the paper on Hypertension Research website (http://www.nature.com/hr) 